\title{
Drug-induced oral mucous membrane pemphigoid: a case report
}

\author{
Bastien Bercault ${ }^{1}$, Mathilde Fenelon ${ }^{1,2}$, Sylvain Catros $^{1,2}$, Jean-Marie Marteau ${ }^{1,{ }^{*}}$, Jean-Christophe Fricain ${ }^{1,2}$ \\ ${ }^{1}$ Head of Odontology and Oral Health, CHU of Bordeaux, France \\ 2 Tissue Bioengineer, Unit 1026, University of Bordeaux, France \\ *Correspondence: bercault787@gmail.com
}

(Received: 29 November 2016, accepted: 1 March 2017)

Keywords:

mucous membrane pemphigoid /

toxidermy

\begin{abstract}
Observation: An 82-year-old patient treated with the antihistamine ebastine (Kestin ${ }^{\oplus}$ ) for allergic rhinitis presented for gingival pain. The diagnosis of mucous membrane pemphigoid was suspected after a positive pinch test. Blood examinations highlighted AC anti-PBGA2, and the gingival biopsy showed an epithelioconjunctival delamination and the presence of anti-IgA antibody and C3 deposition along the basal of the membrane. Topical corticosteroid administration was initiated. The symptoms improved but elevated IgA and C3 levels persisted. He underwent a complete remission on cessation of ebastine treatment. Commentary: Mucous membrane pemphigoid is an autoimmune disease whose origin remains unclear. The aim of the treatment is to decrease the symptoms, but full recovery of the patients is exceptional. We report here a clinical case with symptom resolution because of the ebastine withdrawal. Ebastine can thus be suspected as a cause if a patient presents with oral symptoms of mucous membrane pemphigoid.
\end{abstract}

\section{Observation}

Here we introduce the case of an 82-year-old patient who consulted us in July 2015 for a detached gingival epithelium evolving over a year.

He was receiving various treatments, including the antihistamine ebasti ne $\left(K_{\text {estin }}{ }^{\oplus}\right)$ for a dust-mite allergy, which was continuing over several years.

At the endobuccal clinical examination, this patient had $3-\mathrm{cm}$ long and $5-\mathrm{cm}$ wide bubble lesions on the left mandibular gingivobuccal-jugular furrow in the form of a linear ulceration (Fig. 1). The pinch test was positive (Fig. 2). These lesions were very painful (VAS: 8/10) and not itchy. The lesions were initially unilateral and then became bilateral. The patient had no other lesions. The ophthalmological examination did not reveal any conjunctival lesions.

The patient had initially attempted treatment using antifungal agents, healing ointments, and mouthwashes without any effect. We diagnosed mucous membrane pemphigoid.

Further examinations were performed: a biopsy was performed for histopathological examination and a direct immunofluorescence (IFD) examination as well as enzyme-linked immunosorbent assay (ELISA) tests, for anti-desmoglein 1 and 3 and anti BP180 and BP230.

The pathology result revealed a subepithelial lymphoplasmacytic infiltrate with an epithelial-connective tissue detachment and the presence of the autoimmune antibodies; antiIgG, IgA, and C3 along the basal membrane (Fig. 3). The ELISA test was positive for anti-PBGA2 antibodies.
This confirmed the diagnosis of mucous membrane pemphigoid. Topical administration of clobetasol, applied in the morning and evening for 3 months, was prescribed. This treatment led to a regression of the symptoms, but was not a complete cure.

The patient reported an improvement of the mucosal pemphigoids only 3 weeks after he stopped taking ebastine. During the reassessment at 3 months after presentation, the patient had a total remission of these lesions.

During the 5-month follow-up afterward, the patient did not have any relapses.

The patient was advised to be vigilant with taking medications.

\section{Comments}

Cicatricial pemphigoid (CP) or mucous membrane pemphigoid is part of the autoimmune bullous dermatoses under the skin. It occurs most frequently in the elderly (average age 60-70 years), with women slightly more likely to be affected than men. In France, there are approximately 70 new cases reported every year. However, this incidence is probably underestimated because $C P$ is not an easily identifiable condition.

$\mathrm{CP}$ is characterized by a selective involvement of the mucous membranes, in particular, buccal membranes ( $80-90 \%$ cases). It most often manifests as erosive gingivitis [1], and more rarely of palatal, lingual, or gingival vesicles. Skin damage is inconsistent. Ocular impairment occurs in $60 \%$ cases and produces chronic conjunctivitis and synechia. This is the most serious effect with blindness occurring in $5-20 \%$ cases. 


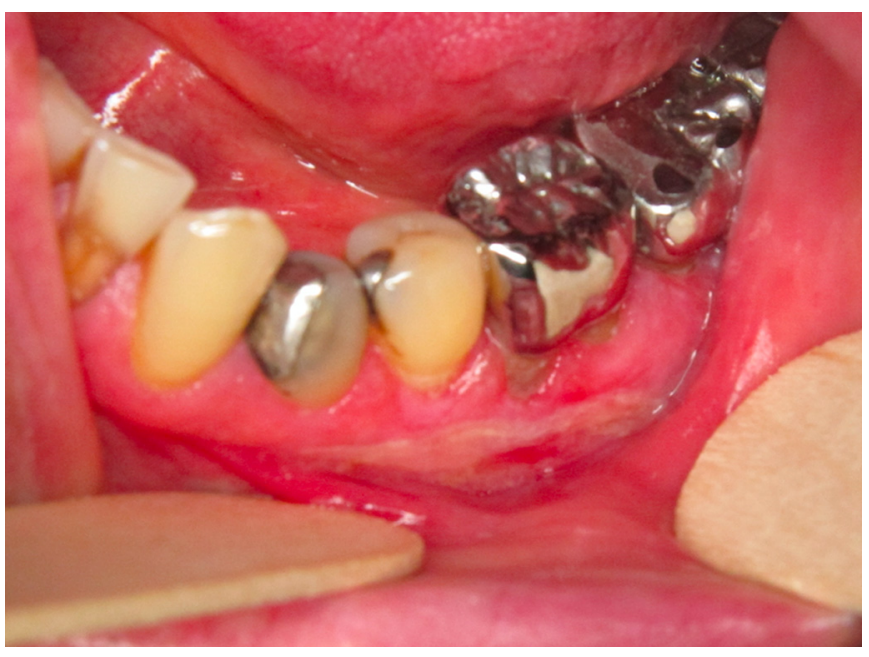

Fig. 1. Bullous lesion in the gingivobuccal-jugal furrow.

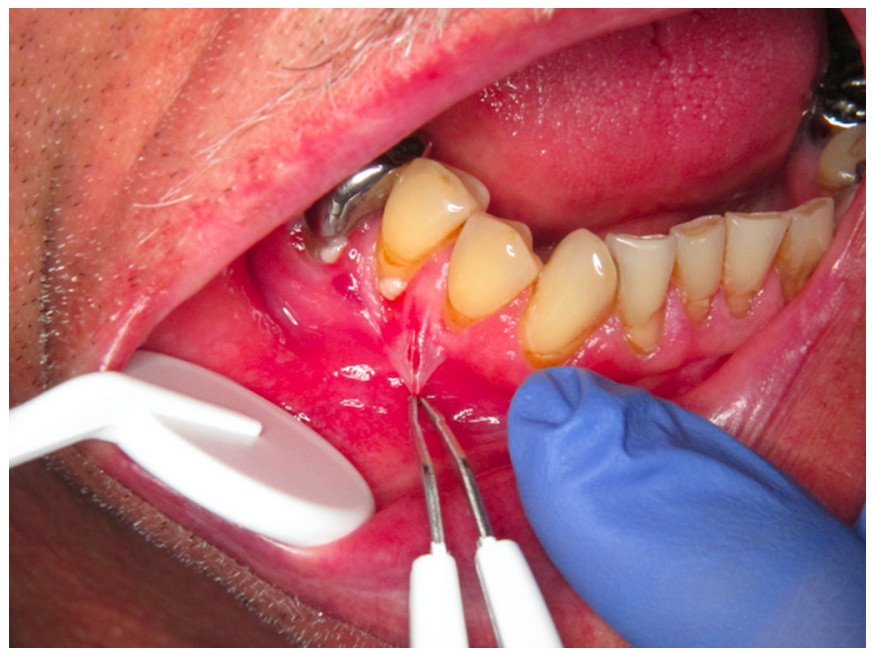

Fig. 2. A positive pinch test.

$\mathrm{CP}$ is characterized by a linear deposition of $\mathrm{C} 3, \mathrm{IgG}$, and/or IgA along the basal membrane [2].

The standard treatment is the application of local corticosteroids immediately and dapsone later [3]. In the event of nonresponse to treatment, oral corticosteroids or immunosuppressive drugs may be prescribed [4].

The causes of this autoimmune disease are still poorly understood, but some medications appear to involve ocular and oral impairment: amlodipine [5], atenolol [6], and furosemide [7]. Previously reported cases suggest the involvement of various medications such as antihypertensive medications (especially those containing a thiol group), diuretics (especially furosemide), antibiotics derived from penicillin, sulfasalazine, phenacetine in the etiology of CP.

Ebastine (Kestin ${ }^{\oplus}$ ) is an antihistamine used to treat allergic rhinitis and urticaria. A statement encouraging people to be

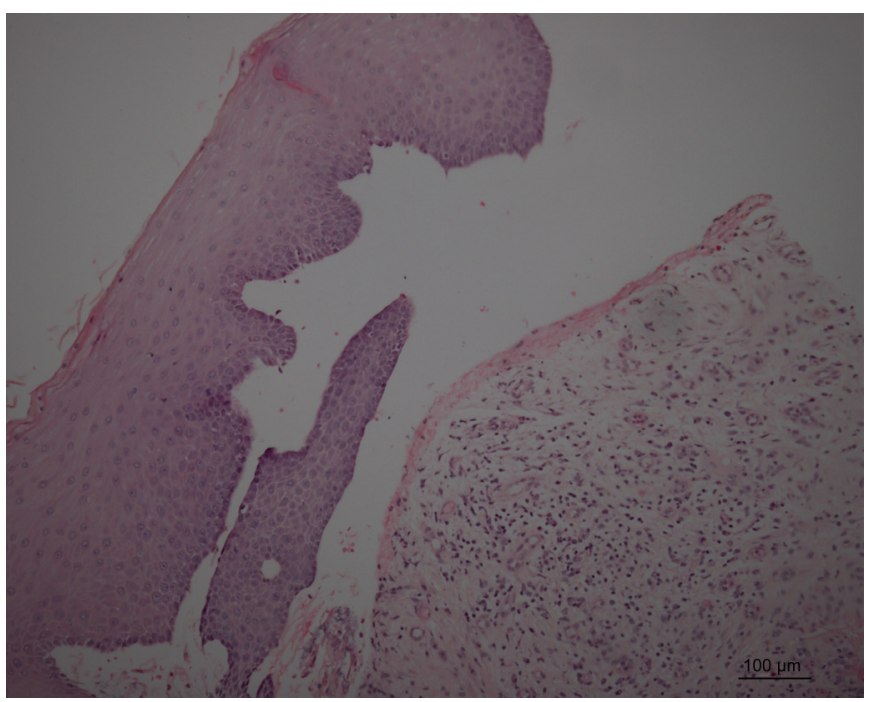

Fig. 3. Histological section: vesicles in the dermoepidermal junction between the epithelium and the basal membrane.

aware of these drugs was made, which confirmed what the literature states regarding the side effects of an adverse drug reaction similar to a CP. However, it is highly likely that this drug in our patient was accountable for the condition. Indeed, the CP completely disappeared 3 months after the patient stopped taking ebastine. No recurrence has been observed in the 6 months after cessation. Usually the clinical signs of CP are diminished by the treatment but the symptoms never disappear entirely. Moreover, the unusual clinical aspect of the lesions, focal, located in the gingivobuccal-jugular furrow is a particular form of CP. Only the reintroduction of the ebastine would have confirmed this diagnosis of a drug-related CP. This table highlights the importance of always looking for possible side effects of medications.

\section{Conflicts of interests}

The authors declare that they has have no conflicts of interest in relation to this article.

\section{References}

1. Vaillant $L$, Chauchaix-Barthès $S$, Hüttenberger $B$, Arbeille $B$, Machet MC, Jan D, Goga D. Le syndrome gingivite erosive chronique : etude retrospective de 33 cas. Ann Dermatol Vénéréol 2000; 127: 381-389.

2. Helander SD, Rogers RS. The sensitivity and specificity of direct immunofluorescence testing in disorders of mucous membranes. $\mathrm{J}$ Am Acad Dermatol 1994; 30: 65-75.

3. Schmidt E, Zillikens D. Pemphigoid diseases. Lancet 2013; 381: 320-332.

4. Chan LS, Ahmed AR, Anhalt GJ et al. The first international consensus on mucous membrane pemphigoid: definition, 


\section{SHORT CASE REPORT}

diagnostic criteria, pathogenic factors, medical treatment and pronostic indicators. Arch Dermatol 2002; 138: 370-379.

5. Williams GS, Goodwin R, Hughes D. Amlopidine as a cause of mucous membrane pemphigoid: first report of amlopidine as a causative agent in MPP. R Eye 2013; 27: 1425.
6. Kanjanabuch P, Arporniem S, Thamrat S, Thumasombut P. Mucous membrane pemphigoid in a patient with hypertension treated atenolol. J Med Case Rep 2012; 6: 373.

7. Lee JJ, D ownham TF. Furosemide-induced bullous pemphigoid: case report and review of literature. J Drugs Dermatol 2006; 5: 562-564. 